\title{
Cytotoxic effects of ultra-diluted remedies on breast cancer cells
}

\author{
MOSHE FRENKEL $^{1}$, BAL MUKUND MISHRA ${ }^{2}$, SUBRATA SEN ${ }^{2}$, PEIYING YANG ${ }^{1}$, ALISON PAWLUS ${ }^{1}$, \\ LUIS VENCE ${ }^{3}$, AIMEE LEBLANC ${ }^{2}$, LORENZO COHEN ${ }^{1}$, PRATIP BANERJI ${ }^{4}$ and PRASANTA BANERJI ${ }^{4}$ \\ ${ }^{1}$ Integrative Medicine Program, ${ }^{2}$ Department of Molecular Pathology, ${ }^{3}$ Department of Melanoma \\ Medical Oncology, The University of Texas M.D. Anderson Cancer Center, Houston, TX, USA; \\ ${ }^{4}$ P. Banerji Homeopathic Research Foundation, Kolkata, India
}

Received May 28, 2009; Accepted July 23, 2009

DOI: 10.3892/ijo_00000512

\begin{abstract}
The use of ultra-diluted natural products in the management of disease and treatment of cancer has generated a lot of interest and controversy. We conducted an in vitro study to determine if products prescribed by a clinic in India have any effect on breast cancer cell lines. We studied four ultra-diluted remedies (Carcinosin, Phytolacca, Conium and Thuja) against two human breast adenocarcinoma cell lines (MCF-7 and MDA-MB-231) and a cell line derived from immortalized normal human mammary epithelial cells (HMLE). The remedies exerted preferential cytotoxic effects against the two breast cancer cell lines, causing cell cycle delay/arrest and apoptosis. These effects were accompanied by altered expression of the cell cycle regulatory proteins, including downregulation of phosphorylated $\mathrm{Rb}$ and upregulation of the CDK inhibitor p27, which were likely responsible for the cell cycle delay/arrest as well as induction of the apoptotic cascade that manifested in the activation of caspase 7 and cleavage of PARP in the treated cells. The findings demonstrate biological activity of these natural products when presented at ultra-diluted doses. Further indepth studies with additional cell lines and animal models are warranted to explore the clinical applicability of these agents.
\end{abstract}

\section{Introduction}

The use of herbs, minerals, vitamins, homeopathic remedies and other complementary and alternative medicine (CAM) is on the rise worldwide, and patients with cancer are increasingly

Correspondence to: Dr Moshe Frenkel, Integrative Medicine Program-Unit 145, The University of Texas M.D. Anderson Cancer Center, 1515 Holcombe Blvd., Houston, TX 77030-4009, USA E-mail: frenkelm@netvision.net.il; moshefrenkelmd@gmail.com

Dr Prasanta Banerji and Dr Pratip Banerji, PBH Research Foundation, 10/3/1 Elgin Road, Kolkata-700 020, India

E-mail: info@pbhrfindia.org; pbhrf@vsnl.com

Key words: breast cancer, breast cancer cells, cell cycle, apoptosis, homeopathy, cancer care, alternative medicine, complementary medicine, integrative medicine, integrative oncology opting to be treated with CAM therapeutic regimens (1-3). The safety and efficacy of many CAM approaches have not been well studied, especially in cancer care. Therefore, the US National Cancer Institute (NCI) developed the Best Case Series program inviting CAM practitioners worldwide to present their clinical experience and 'best cases' in the use of alternative medicine in the treatment of cancer, with the objective to develop further research toward rigorous scientific validation.

In 1999, the NCI evaluated a cancer treatment protocol developed at the P. Banerji Homeopathic Research Foundation (PBHRF) in Kolkata, India. The 'Banerji protocol' used specific ultra-diluted natural substances to treat patients with different cancers. The NCI reviewed 10 patients treated on the Banerji protocol. In four of the cases with lung and esophageal cancers, the NCI confirmed partial responses (4). All patients reviewed had appropriate pathology and imaging studies to confirm diagnosis and response. The patients only received the remedies prescribed at the PBHRF clinic and did not receive any additional conventional treatment, such as surgery, radiation, or chemotherapy. After rigorous evaluation, the NCI concluded that there was sufficient evidence of efficacy to warrant further research of the Banerji protocol.

As documented by the clinic, 21,888 patients with malignant tumors who were treated only on the Banerji protocol were followed at PBHRF between 1990 and 2005. Of the patients, 941 had breast cancer. Clinic physicians reported that in $19 \%$ of the patients, the malignant tumors completely regressed, and in $21 \%$ the tumors were stable or improved with treatment. For patients with stable tumors, follow-up continued for at least 2 years and for as long as 10 years (5).

In 2003, Pathak et al reported that an ultra-diluted dose of the homeopathic remedy Ruta graveolens, commonly prescribed as the standard Banerji protocol therapeutic agent for brain cancer, selectively induced death in glioblastoma multiforme cells while promoting the proliferation of normal peripheral blood lymphocytes (6).

Since those findings were reported, we have noticed that patients who come to M.D. Anderson's Integrative Medicine Clinic already use homeopathy or have a marked interest in integrating this treatment with their conventional therapies because the agents have no toxicity and are easy to use. Most of the information on the use of these agents is available to patients on the Internet and through support groups, and 
many of the remedies can be purchased at health food stores, supermarkets, or from online vendors.

Considering the growing interest in Banerji protocol remedies among patients at our clinic, most of whom have advanced breast cancer, we decided to evaluate the in vitro effect of those remedies. In this study, we compared the effects of four ultra-diluted remedies in two well-characterized human breast cancer cell lines and an immortalized normal human mammary epithelial cell line grown in vitro.

\section{Materials and methods}

Drugs. The ultra-diluted remedies used by PBHRF were obtained from Sharda Boiron Laboratories Ltd India and additional similar remedies obtained from the Standard Homeopathic Company (King of Prussia, PA, USA). The experiments were conducted in triplicate and repeated at least twice in each case of remedy. The remedies used and their dilutions were the same as those prescribed by the PBHRF for the treatment of human breast cancer: Carcinosin, 30C; Conium maculatum, 3C; Phytolacca decandra, 200C and Thuja occidentalis, 30C. The remedies were diluted with $87 \%$ extra-neutral alcohol, referred to as the 'solvent'. The solvent was also evaluated for its effects on the cells both by itself and after being processed through a process of succussion method similar to the way used for making the potency dilutions of the remedies.

High-performance liquid chromatography (HPLC) for detection of chemical components in ultra-diluted remedies. Ultra-diluted remedies may contain many chemical constituents, since they are derived from plant and animal cell extracts. Fingerprinting of the remedies was therefore performed by HPLC to identify constituents. Fingerprinting was performed with Waters Delta 600 HPLC systems (Waters Co., Milford, MA) equipped with a solvent delivery pump unit, a Waters 717 plus autosampler, and a Waters 2996 photodiode array detector. Chromatographic separation was achieved using a C18 column $(5.0 \mu \mathrm{m}, 250 \times 4.6 \mathrm{~mm})$ (Agilent) with a guard cartridge $(5.0 \mu \mathrm{m}, 20 \times 3.9 \mathrm{~mm}$ I.D.). The mobile phase consisted of methanol (A) and water (B), and separation was achieved using a linear gradient of 10-90\% methanol with an injection-to-injection time of $60 \mathrm{~min}$. The flow rate was $1 \mathrm{ml} / \mathrm{min}$. The detection wavelengths were 254 and $220 \mathrm{~nm}$. All samples were run under the same conditions and had the same scale of 0.2 astronomical units.

Cell lines. Two human breast adenocarcinoma cell lines, MCF-7 and MDA-MB-231, were obtained from the American Tissue Culture Collection (Manassas, VA). The MCF-7 cells harbor the wild-type p53 gene and are estrogen- and progesterone-receptor positive, while the MDA-MB-231 cells harbor a mutant p53 gene and are estrogen- and progesterone-receptor negative. The cells were cultured in Dulbecco's modified Eagle's medium supplemented with $10 \%$ heat-inactivated fetal bovine serum (Atlanta Biologicals, Atlanta, GA), 1\% L-glutamine (Invitrogen, Carlsbad, CA) and $1 \%$ pen-strep (Invitrogen). A control cell line, HMLE, which was derived form normal human mammary epithelial cells immortalized with the catalytic subunit of telomerase and SV40 large-T and small-T antigens, was kindly provided by Dr Sendurai Mani of M.D. Anderson. The HMLE cells were cultured in a 1:1 ratio of Dulbecco's modified Eagle's medium: Ham's F12 (Mediatech, Manassas, VA) and Mammary Epithelial Cell Growth Medium (Lonza, Allendale, $\mathrm{NJ})$; the culture was supplemented with $2.5 \mu \mathrm{g} / \mathrm{ml}$ insulin (Sigma), $5 \mathrm{ng} / \mathrm{ml}$ human Epidermal Growth Factor (Sigma), and $250 \mathrm{ng} / \mathrm{ml}$ hydrocortisone (Sigma). All the cell lines were incubated at $37^{\circ} \mathrm{C}$ in an atmosphere of $5 \% \mathrm{CO}_{2}$ and $95 \%$ air.

Methylthiazolyldiphenyl-tetrazolium bromide (MTT) assay. Cell lines were analyzed for cell viability by MTT assay. Approximately 5,000 cells grown in triplicate in 96-well plates for $24 \mathrm{~h}$ were treated with the remedies for $24,48,72$ and $96 \mathrm{~h}$. To determine cell viability, $50 \mu \mathrm{l}$ of MTT $(5 \mathrm{mg} / \mathrm{ml})$ was added in the dark in sterile conditions to the cells for $2 \mathrm{~h}$ at $37^{\circ} \mathrm{C}$. After $2 \mathrm{~h}$, the medium was removed and $100 \mu \mathrm{l}$ dimethyl sulfoxide was added. The plates were incubated at room temperature for 8-10 min. The absorbance at OD $570 \mathrm{~nm}$ was then measured by an enzyme-linked immunosorbent assay plate reader.

Cytologic preparations and fluorescence in situ hybridization (FISH). Remedy-treated and control cell cultures were treated with $0.04 \mu \mathrm{l} / \mathrm{ml}$ of colcemid (Gibco/Invitrogen, Carlsbad, CA) for about an hour at $37^{\circ} \mathrm{C}$ and then processed for chromosome preparation following a brief hypotonic treatment in $0.9 \%$ sodium citrate and fixation in a $3: 1$ solution of methanol and acetic acid. The slides were air-dried and stained with Giemsa stain, and the cultures were evaluated for their mitotic indices. The slides were also processed for FISH using a Cy-3-labeled peptide nucleic acid telomeric probe (Dako Corporation, Carpinteria, CA) according to the manufacturer's protocol. At least 200 cells from treated and untreated samples were analyzed for mitotic index and telomeric DNA signals with a Nikon Eclipse 80i microscope equipped with fluorescence attachment and a Photometrics CoolSNAP HQ2 monochrome digital camera.

Flow cytometry for cell cycle distribution and subdiploid population assay. The cell cycle progression and apoptosis were analyzed by fluorescence activated cell sorting (FACS) analysis. Approximately $1 \times 10^{6}$ each of the control and remedy-treated cells were trypsinized, washed twice with cold phosphate-buffered saline (1X PBS) and fixed in $2 \mathrm{ml}$ of ice-cold ethanol $(70 \%)$ overnight at $4^{\circ} \mathrm{C}$. Fixed cells were washed twice with $1 \mathrm{X}$ PBS and then incubated with $1 \mathrm{ml}$ of PBS containing $20 \mu \mathrm{g} / \mathrm{ml}$ RNAse and $50 \mu \mathrm{g} / \mathrm{ml}$ propidium iodide (PI) for $30 \mathrm{~min}$ at $37^{\circ} \mathrm{C}$. The stained cells were analyzed using a Coulter Epics XL cell counter (Beckman Coulter, Brea, CA).

Apoptosis detection by annexin V labeling and flow cytometry. Remedy-treated and control cells $\left(\sim 1 \times 10^{6}\right.$ cells each) were cultured in $60 \mathrm{~mm}$ plates for $72 \mathrm{~h}$. Both floating and adherent cells were collected by centrifugation at 1,200 rpm for $5 \mathrm{~min}$ at $4^{\circ} \mathrm{C}$. The cell pellet was washed once with cold $1 \mathrm{X}$ PBS. The pellet was re-suspended in $500 \mu \mathrm{l}$ of $\mathrm{TBS} / \mathrm{Ca}^{2+}[20 \mathrm{mM}$ Tris plus $150 \mathrm{mM} \mathrm{NaCl}(\mathrm{pH} 7.4)$ and $2 \mathrm{mM} \mathrm{Ca}^{2+}$. Then, $25 \mu \mathrm{l}$ of annexin V/PI solution (Trevigen, Gaithersburg, MD) was 
added into the solution and incubated for $5 \mathrm{~min}$ at room temperature. The reaction was analyzed by FACS at an emission wavelength of about $525 \mathrm{~nm}$ with a blue laser for annexin V-fluorescein isothiocyanate (FITC) staining and at an emission wavelength of about $620 \mathrm{~nm}$ with a red laser for PI staining.

Immunoblotting. Expression of proteins associated with cell cycle regulation and apoptosis was detected by Western blot analysis of the control and remedy-treated cells. For cell cycle analysis, we used antibodies against the cell cycle regulatory proteins cyclin D1, cyclin D3, cyclin-dependent kinase 4 (CDK4), cyclin-dependent kinase 6 (CDK6), p27 (a $\mathrm{CDK}$ inhibitor) and the phosphorylated form of $\mathrm{Rb}$ protein while to analyze apoptotic response, antibodies against the apoptosis-associated proteins poly (ADP-ribose) polymerase (PARP) and caspase 7 (Cell Signaling Technology, Danvers, MA) were used. To isolate total proteins, cell pellets were washed once with ice-cold $1 \mathrm{X}$ PBS, and then resuspended in $100 \mu \mathrm{l}$ of cold lysis buffer (20 mM Tris- $\mathrm{HCl}, \mathrm{pH} 8.0,150 \mathrm{mM}$ $\mathrm{NaCl}, 0.1 \mathrm{mM}$ phenylmethylsulfonyl fluoride $1 \mathrm{mM}$ ethylene diamine tertaacetic acid, $1 \%$ Triton $\mathrm{X}-100,0.5 \%$ sodium deoxycholate, $0.1 \%$ sodium dodecyl sulfate (SDS), $0.5 \mathrm{mg}$ of complete Protease inhibitor cocktail), homogenized and centrifuged. Protein concentrations were determined using a Bio-Rad (Richmond, CA) protein assay kit. Equal amounts of total cellular protein $(50 \mu \mathrm{g})$ were suspended in $1 \mathrm{X}$ sample buffer (125 mM Tris-HCl (pH 6.8), 2\% SDS, 5\% glycerol, $0.1 \%$ bromophenol blue and $1 \%$ B-mercaptoethanol) and denatured by boiling for $5 \mathrm{~min}$. The prepared samples were resolved by $10 \%$ SDS-polyacrylamide gel electrophoresis and transferred onto a Hybond-ECL nitrocellulose membrane (Amersham Biosciences, Piscataway, NJ). The membranes were then incubated with the desired antibodies overnight at $4^{\circ} \mathrm{C}$. The membranes were subsequently incubated for $1 \mathrm{~h}$ with anti-rabbit immunoglobulin $\mathrm{G}$ antibody conjugated to horseradish peroxidase and visualized using enhanced chemiluminescence kits (GE Healthcare, Bucks, UK).

\section{Results}

Chromatographic fingerprinting. The solvent showed only one distinct peak, eluted at about $2 \mathrm{~min}$, in the chromatogram. To determine whether succussion caused any chemical changes in the solvent, we compared the fingerprinting profiles of the solvent before and after succussion. The chromatogram of the untreated and treated solvents appeared identical, indicating that succussion did not cause chemical changes in the solvent. All four remedies had very similar HPLC chromatograms to each other, with only trace amounts of limited number of peaks. They were not significantly distinct from the solvent and they lacked the distinct peak seen in the solvent.

Ultra-diluted remedies reduce viability of human breast adenocarcinoma cells. As shown in Fig. 1A, the solvent reduced the viability of all three cell types; the overall reduction in cells at different doses of solvent was about $30 \%$ for MCF-7, 20-30\% for MDA-MB-231 and 20\% for HMLE cells. Interestingly, the inhibitory effects on cell viability of the remedies in both the MCF-7 and MDA-MB-231 cells were distinctly greater for each of the doses tested than those seen in cells treated only with solvent. MCF-7 cells were found to be more sensitive to all four remedies than the MDA-MB-231 cells (Fig. 1A, panels 2 and 3). The inhibitory effects of the remedies were also dose-dependent for both MCF-7 and MDA-MB-231 cells, with progressively more inhibition seen at higher doses (50-75\% loss of viability in the two cell lines). To investigate whether the inhibitory effects increased with time of treatment, we treated the cells with the solvent and remedies at concentrations of 5 and $10 \mu \mathrm{l} / \mathrm{ml}$ for 48 and $72 \mathrm{~h}$. The results revealed that the inhibitory effects were higher for the longer period of treatment and greater inhibition was caused by the remedies than by the solvent. Among the four remedies investigated, Carcinosin and Phytolacca, reproducibly revealed relatively higher inhibitory effects in replicate experiments. These two remedies reduced viability of the MCF-7 cells by $60-75 \%$ at $5 \mu \mathrm{l} / \mathrm{ml}$ and by $70-80 \%$ at $10 \mu \mathrm{l} / \mathrm{ml}$ doses after 48 and $72 \mathrm{~h}$ treatments, respectively. For the MDA-MB-231 cells, the reductions were $50-65 \%$ at $5 \mu \mathrm{l} / \mathrm{ml}$ and $65-70 \%$ at $10 \mu \mathrm{l} / \mathrm{ml}$ at these times. The solvent, on the other hand, caused reduction in survival of the two cell lines by $30-35 \%$ under the same conditions (Fig. 1B). Strikingly, the effects of the four remedies on the viability of the HMLE cells were nearly indistinguishable from the effects of the solvent alone (Fig. 1A and B). In view of the fact that Carcinosin and Phytolacca appeared relatively more potent inhibitors of cancer cell survival, we decided to investigate these two remedies further to understand the mechanisms responsible for their observed effects on cell proliferation and survival.

Induction of cell cycle arrest and cell death by the remedies. We next examined cell cycle progression profiles of MCF-7, MDA-MB-231 and HMLE cells treated with $5 \mu \mathrm{l} / \mathrm{ml}$ dose of Carcinosin and Phytolacca. As shown in Fig. 2A, at $24 \mathrm{~h}$ of treatment with the remedies, MCF-7 cells showed G1 phase arrest with a concomitant decrease in the $\mathrm{S}$ and $\mathrm{G} 2 / \mathrm{M}$ phase populations. At $48 \mathrm{~h}$ and later, distinct sub-G0 populations, representing cell death, appeared and G1 phase cells decreased. The sub-G0 population showed a marginal increase at $96 \mathrm{~h}$ post-treatment, while $S$ phase cells had increased and G2/M phase cells were almost completely absent. In the HMLE cells, a relatively less pronounced G1 phase arrest was evident after $24 \mathrm{~h}$ of treatment with both the solvent and the remedies, while a relatively greater $\mathrm{G} 2 / \mathrm{M}$ phase delay or arrest was seen following treatment with the remedies than with the solvent treated and untreated cells. A sub-G0 population became evident at 72 and $96 \mathrm{~h}$ in the treated samples, though the peak was distinctly smaller than in the remedy-treated MCF-7 cells. The cell cycle profiles of the remedy-treated MDA-MB-231 cells appeared different from those of the remedy-treated MCF-7 cells. While a G1 arrest in cells treated for $24 \mathrm{~h}$ with solvent and Carcinosin was similar to that seen in HMLE cells, the same was not observed after Phytolacca treatment. The Phytolacca-treated cells had greater G2/M phase accumulation following 24, 48 and $72 \mathrm{~h}$ of treatment than the MCF-7 cells. Sub-G0 peaks were visible at 48,72 and $96 \mathrm{~h}$, but those peaks were not seen in the untreated controls and were higher than those seen in the 
A
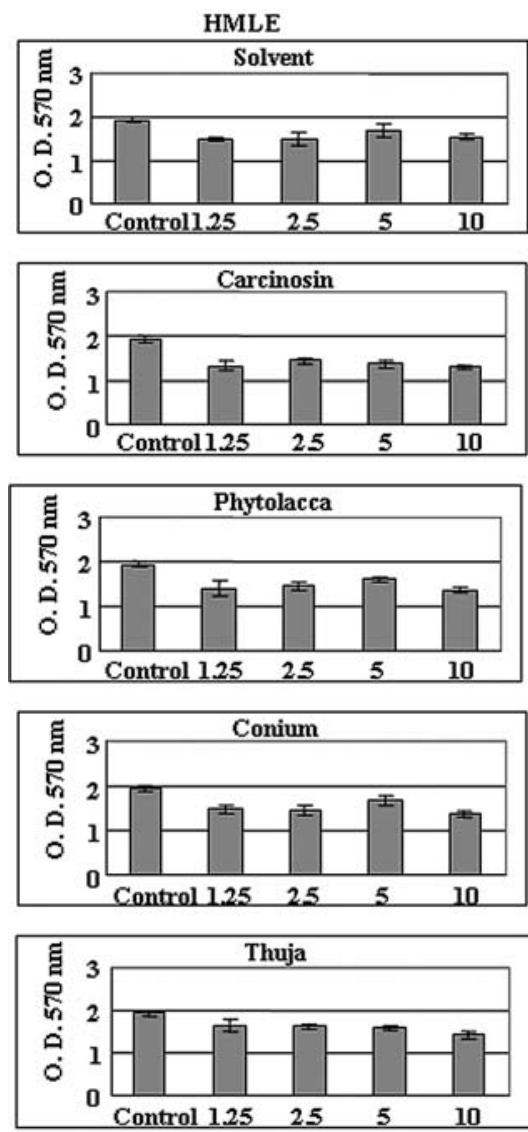

MCF-7
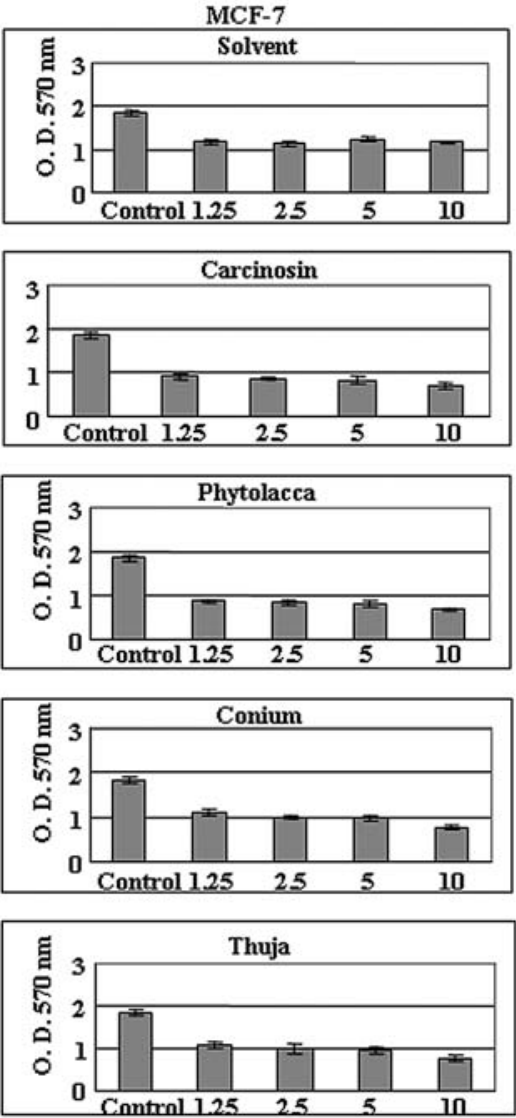

MDA-MB-231
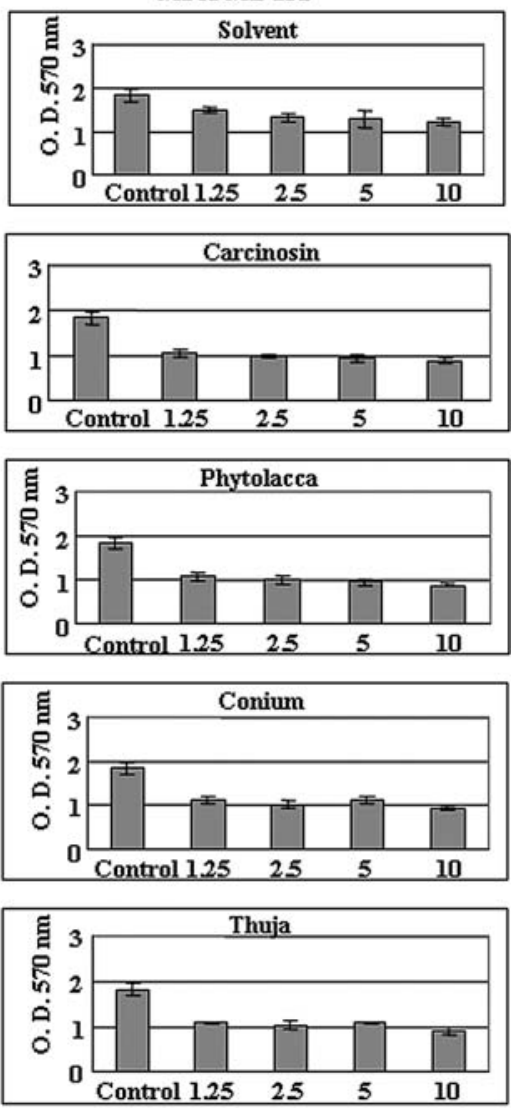

B
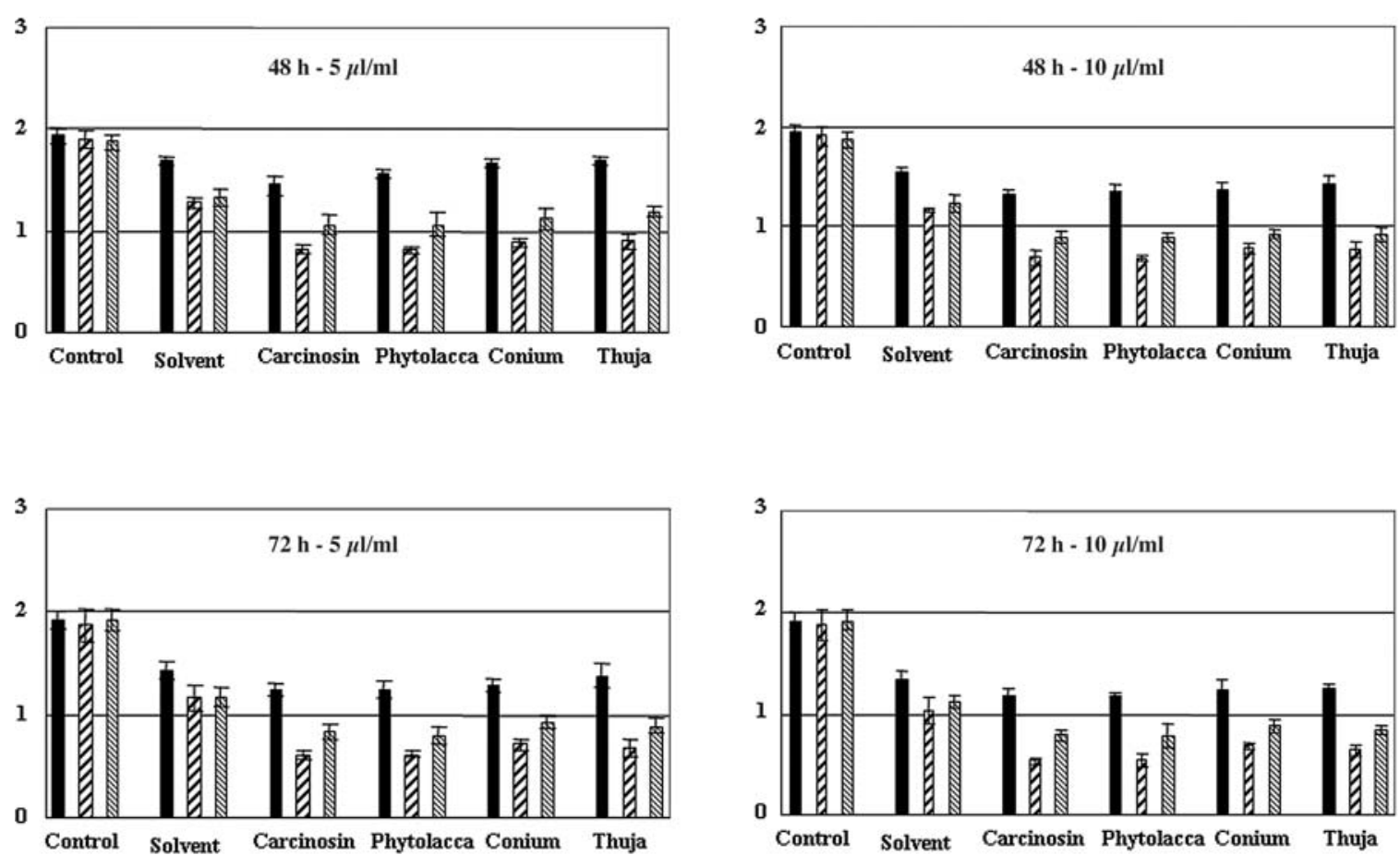

$\square$ HMLE MCF-7

Figure 1. (A) Methylthiazolyldiphenyl-tetrazolium (MTT) bromide viability assay of HMLE, MCF-7 and MDA-MB-231 cells following treatments with the solvent, Carcinosin, Phytolacca, Conium, or Thuja for $48 \mathrm{~h}$ at various concentrations $(0,1.25,2.5,5$ and $10 \mu 1 / \mathrm{ml})$. The values were obtained in independent experiments performed in triplicate and were represented as mean \pm standard error vs. control (bars). (B) MTT assay for viability of the cells treated with 5 and $10 \mu \mathrm{l} / \mathrm{ml}$ solvent, Carcinosin, Phytolacca, Conium, or Thuja for 48 and $72 \mathrm{~h}$. The values were obtained in independent experiments performed in triplicate and are presented as mean \pm standard error (bars) vs. control. 
A
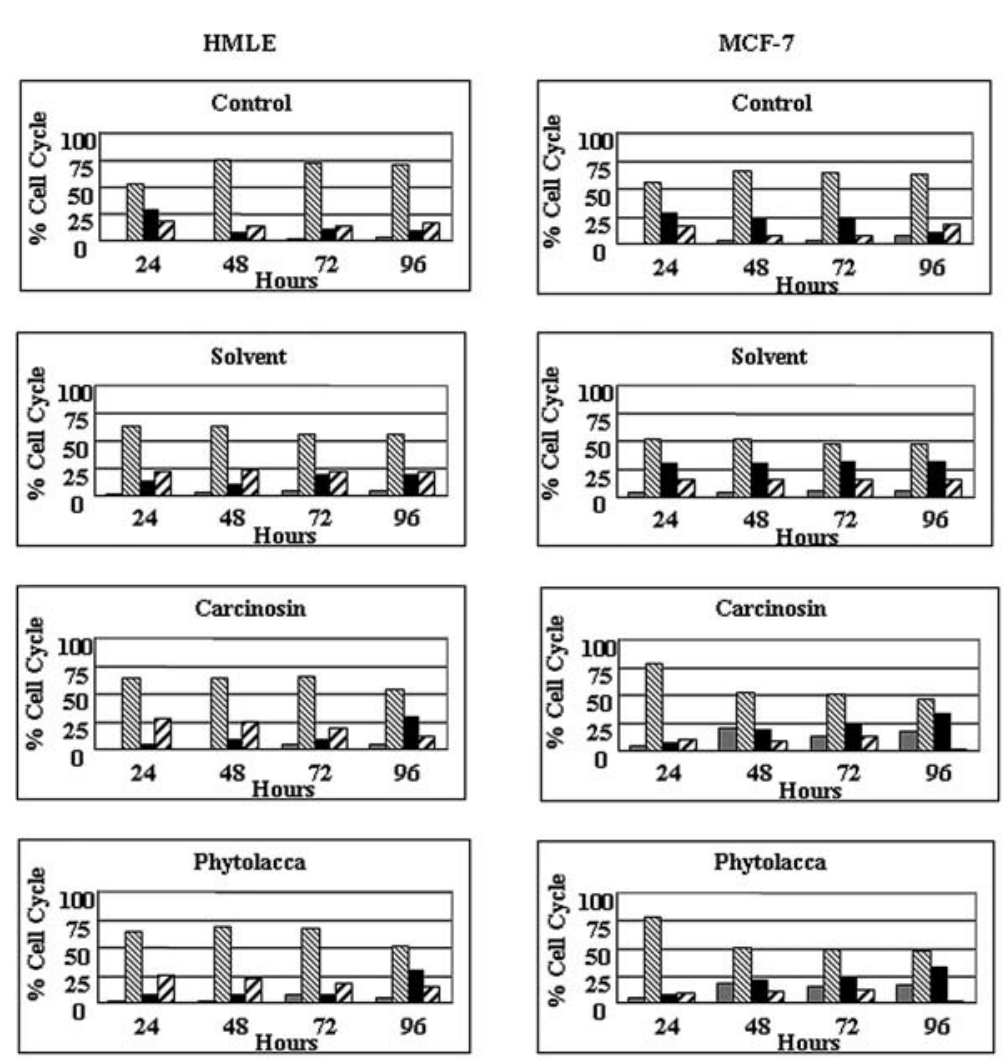

Sub Go/Gl

Gl

B

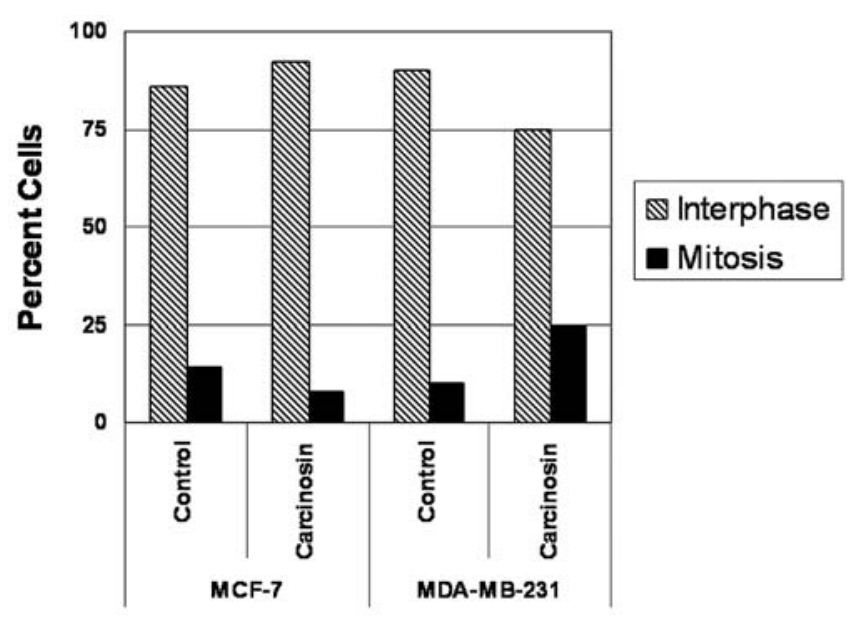

solvent-treated samples. Preferential growth delay or arrest of the MDA-MB-231 cells at the G2/M phase following treatment with the remedies was revealed with microscopic analysis of the remedy-treated cells. The number of mitotic cells was clearly elevated in the treated MDA-MB-231 cells compared to the treated MCF-7 cells and the untreated controls (Fig. 2B).

Preferential loss of telomeric DNA in remedy-treated adenocarcinoma cells. The MCF-7, MDA-MB-231 and HMLE cells treated with Carcinosin and Phytolacca were analyzed by FISH to quantify telomeric DNA. As shown in Fig. 3, there was significant reduction in telomeric DNA signals in the remedy-treated cells compared to the untreated controls. The
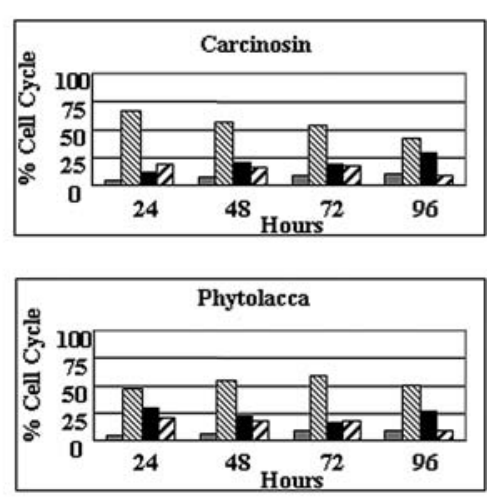

MDA-MB-231
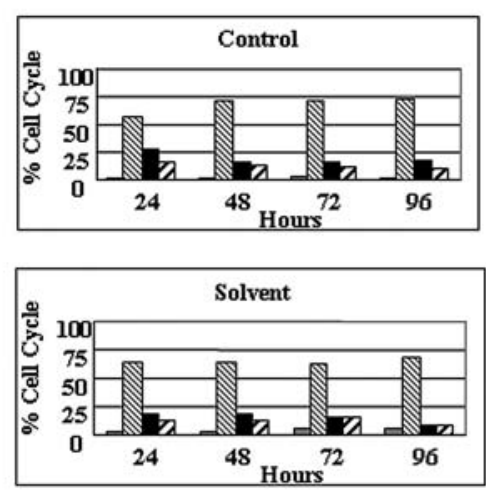

G2
Figure 2. (A) Cell cycle progression analyses of the HMLE, MCF-7 and MDA-MB-231 cells treated with $5 \mu 1 / \mathrm{ml}$ solvent, Carcinosin or Phytolacca for various time points $(24,48,72$ and $96 \mathrm{~h})$. Fluorescence-activated cell sorting (FACS) was used to develop a DNA histogram and determine the percentages of cells in each cell cycle phase. (B) Mitotic indices of control and Carcinosin-treated MCF-7 and MDA-MB-231 cells.

telomeric DNA signals in the treated HMLE cells were much less affected than signals in the adenocarcinoma cells. This preferential reduction of telomeric DNA signals indicated that Carcinosin and Phytolacca caused loss of genomic integrity, which possibly contributed to induction of cell death.

Altered expression profiles of the cell cycle regulatory proteins in Carcinosin- and Phytolacca-treated cells. Since the cells treated with the remedies displayed cell cycle delay/arrest, we analyzed the expression profiles of the cell cycle regulatory proteins in Carcinosin- and Phytolacca-treated HMLE, MCF-7 and MDA-MB-231 cells (Fig. 4). The cell cycle proteins analyzed in these experiments included cyclins D1 and D3, CDK4, CDK6, phosphorylated Rb (pRb) and p27. Each of the three cell types revealed both unique and common changes in the expression profiles of the cell cycle regulatory proteins, reflecting the cycle pathway-associated molecular alterations that occurred in response to the remedy treatments. In the HMLE cells, the levels of cyclins D1 and D3 decreased significantly, but those cyclins did not show any perceptible change in expression in the MCF-7 and MDAMB-231 cells. The levels of CDK4 and CDK6 declined at $24 \mathrm{~h}$ and then were marginally elevated at later time points in the 

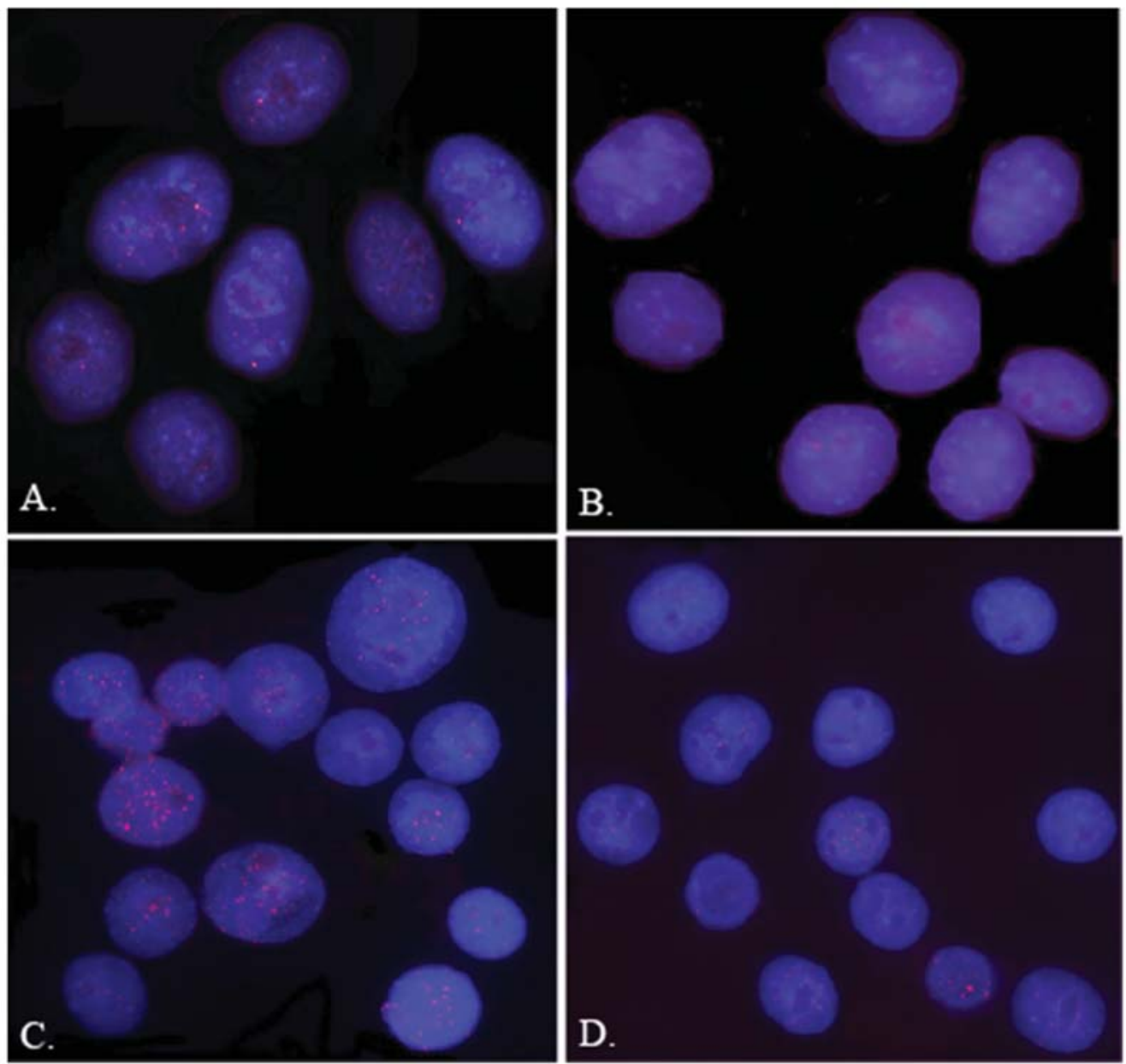

Figure 3. (A) Representative telomere DNA fluorescence in situ hybridization images of untreated MCF-7 cells. (B) MCF-7 cells treated with Carcinosin. (C) Untreated MDA-231 cells. (D) MDA-MB-231 cells treated with Carcinosin. Treated cells were exposed to $5 \mu 1 / \mathrm{ml}$ Carcinosin for $72 \mathrm{~h}$.

HMLE

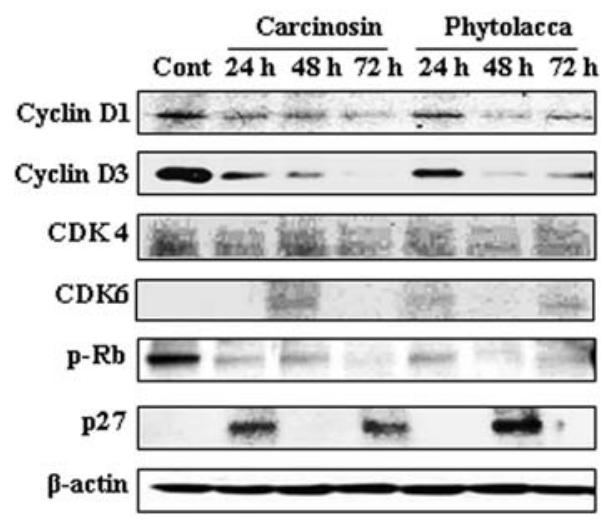

MCF-7

Carcinosin Phytolacca
MDA-MB-231

Carcinosin Phytolacca

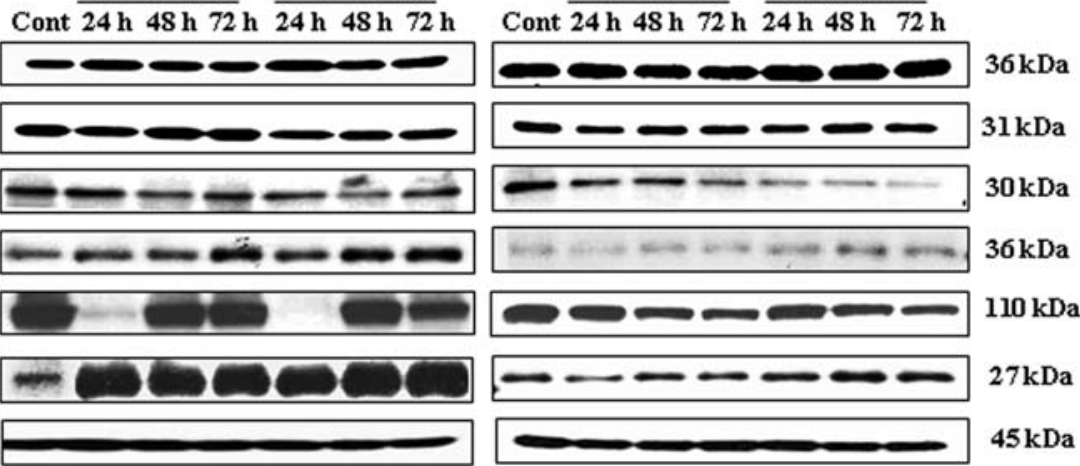

Figure 4. Western blot analyses of cell cycle regulatory proteins in HMLE, MCF-7 and MDA-MB-231 cells, treated with $5 \mu 1 / \mathrm{ml}$ Carcinosin or Phytolacca for various lengths of time $(24,48$ and $72 \mathrm{~h})$. Protein loading was verified by detection of $\beta$-actin in the same gels.

HMLE cells. The lowered expression levels of the CDKs coincided with elevated levels of p27 and lowered levels of $\mathrm{pRb}$, likely reflecting the mechanism of cell cycle delay/ arrest in these cells. In the MCF-7 and MDA-MB-231 cells, while the levels of cyclins D1 and D3 remained almost unchanged in the treated cells, the levels of CDK4 and CDK6 showed contrasting expression patterns following remedy treatments. In MCF-7 cells, CDK4 and CDK6 remained elevated at $24 \mathrm{~h}$, had decreased at $48 \mathrm{~h}$, and had increased at $72 \mathrm{~h}$, whereas the levels showed a gradual decline in MDAMB-231 cells. These minimal changes in the G1 phase CDKs in MCF-7 cells coincided with their delay/arrest at this cell cycle stage, and their decline in MDA-MB-231 cells reflected uninhibited progression through this phase of the cell cycle. 
Control

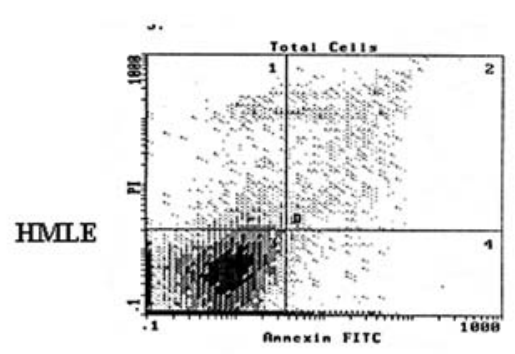

MCF-7
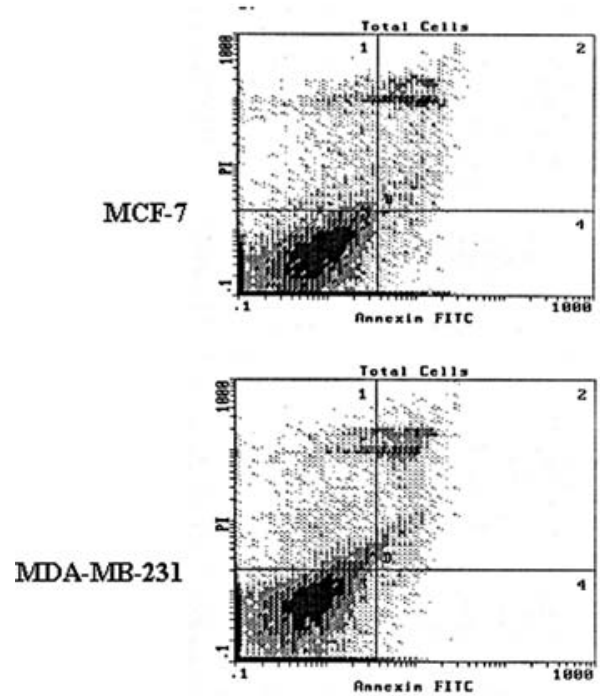

Carcinosin
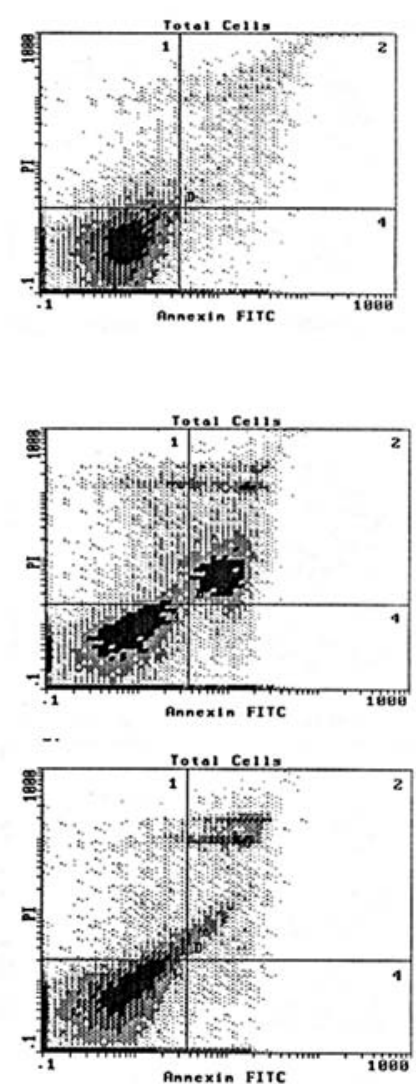

Phytolacca
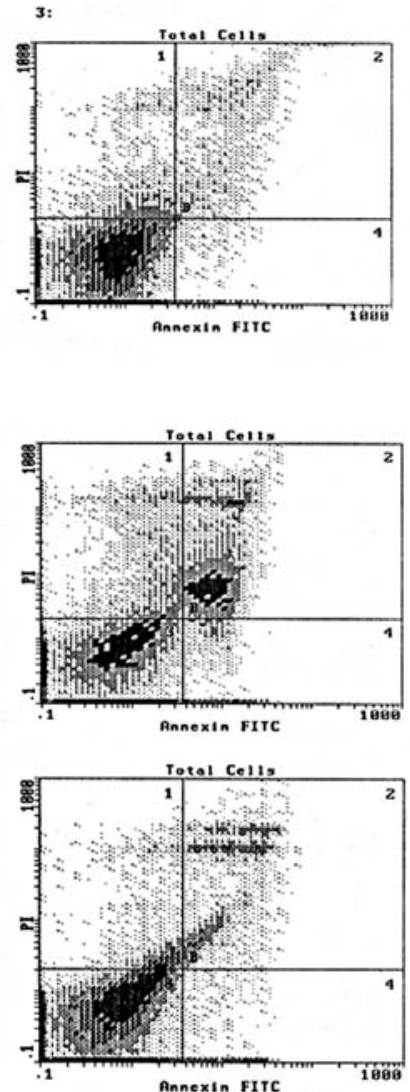

Figure 5. Density plot of apoptotic response in HMLE, MCF-7 and MDA-MB-231 cells treated with $5 \mu 1 / \mathrm{ml}$ Carcinosin or Phytolacca for 72 h. Apoptotic response was determined by fluorescence-activated cell sorting. Viable cells are in the lower left quadrant, and early- and late-stage apoptotic cells are in the lower right and upper right quadrants. Note the increase in late apoptotic MCF-7 and MDA-MB-231 cells.

Interestingly, despite maintenance of the elevated CDK4 and CDK6 levels at $24 \mathrm{~h}$ in the treated MCF-7 cells, the level of its phosphorylated substrate, $\mathrm{Rb}$, showed a sharp decline at $24 \mathrm{~h}$. This coincided with elevated expression of $\mathrm{p} 27$, indicating that induction of p27 following remedy treatment played an important role in regulating the proliferation and viability of these MCF-7 cells through the cell cycle. A progressive decline of $\mathrm{pRB}$ in MDA-MB-231 cells from 24 to $72 \mathrm{~h}$ of remedy treatment, on the other hand, demonstrated that the remedies had minimal effect during transition through the G1 phase of these cells. However, the increasing level of p27 through $72 \mathrm{~h}$ of treatment suggested that induction of this kinase inhibitor is responsible for eliciting the cytostatic and cytotoxic responses to the remedies in MDA-MB-231 cells.

Induction of apoptosis and activation of the apoptotic cascade in Carcinosin and Phytolacca-treated cells. To confirm that the ultra-diluted remedies induced apoptosis, HMLE, MCF-7 and MDA-MB-231 cells treated with Carcinosin and Phytolacca for $72 \mathrm{~h}$ were stained with PI and annexin V-Alexa Fluor 488 and analyzed with FACS.

Fig. 5 shows that Carcinosin and Phytolacca markedly increased the late apoptotic population, as evidenced by the increased number of MCF-7 cells in the upper right quadrant of the FACS histogram; late apoptosis was also higher in
MDA-MB-231 than in the controls. In contrast, the numbers of such late apoptotic cells were only marginally elevated in the HMLE cells. Quantitatively, Carcinosin and Phytolacca increased the population of apoptotic cells only from 5 to $10 \%$ in solvent-treated control cells. The increase in the apoptotic MCF-7 cell population was much larger, ranging from $7 \%$ in solvent-treated cells to $27 \%$ and $28 \%$, respectively, in Carcinosin- and Phytolacca-treated cells. The increase in the apoptotic cell population in Carcinosin- and Phytolaccatreated MDA-MB-231 cells was less pronounced, ranging from $10 \%$ in control cells to 18 and $16 \%$, respectively, in Carcinosin- and Phytolacca-treated cells.

As initiation of the apoptotic cascade is associated with the generation of activated cleaved caspases as well as cleavage of PARP, we analyzed these molecular markers of apoptosis in the Carcinosin- and Phytolacca-treated HMLE, MCF-7 and MDA-MB-231 cells. As shown in Fig. 6, treatment of MCF-7 cells enhanced PARP degradation, resulting in the appearance of the $85-\mathrm{kDa}$ PARP fragment in Western blot analyses. Surprisingly, in the MDA-MB-231 cells, the amount of intact PARP progressively declined with increasing time of treatment but the cleaved $85-\mathrm{kDa}$ band was not evident. HMLE cells showed minimal cleavage of PARP after treatment with the remedies. Furthermore, activation of the apoptotic cascade in the remedy-treated cells was evident from the appearance of the activated cleaved caspase 7 in both 


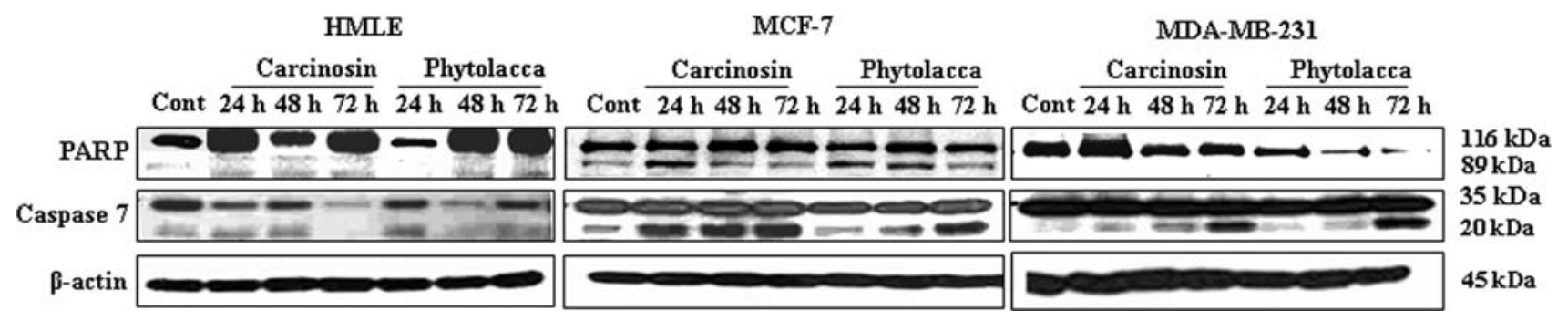

Figure 6. Western blot analyses of poly (ADP-ribose) polymerase cleavage and activated cleaved caspase 7 in Carcinosin- and Phytolacca-treated HMLE, MCF-7 and MDA-MB-231 cells. Protein loading in the gel was verified with the detection of $\beta$-actin.

the MCF-7 and MDA-MB-231 cells. Whereas activation of caspase 7 was minimal in the treated HMLE cells, the activated form of the enzyme was evident from $24 \mathrm{~h}$ onward during Carcinosin treatment and at $72 \mathrm{~h}$ of Phytolacca treatment in the MCF-7 cells. Activation of caspase 7 in MDA-MB-231 cells, on the other hand, was clearly detectable after $72 \mathrm{~h}$ of treatment with Carcinosin and Phytolacca. The results therefore demonstrate that apoptotic cascade was preferentially activated in the remedy-treated carcinoma cells compared to the normal cells and that the activation in the cancer cells was temporally regulated in a cell-type-specific manner.

\section{Discussion}

Our findings suggest that ultra-diluted homeopathic remedies prescribed in the 'Banerji protocol' exert preferential cytotoxic effects against the human breast carcinoma cell lines MCF-7 and MDA-MB-231. Further, we found that these effects resulted from altered expression of cell cycle regulatory proteins, which causes cell cycle delay/arrest as well as induction of cell death by activation of the apoptotic cascade. Preferential inhibition of survival and induction of cell death in presence of the remedies compared with the solvent and also the dose-dependence of these effects validate our conclusion. Importantly, the study also revealed that these remedies have relatively less toxic effects on the survival of cells derived from normal mammary epithelium and moderately inhibits proliferation of peripheral blood mononuclear cells (PBMC). Proliferation of CD3 and IL-2 stimulated PBMCs, assayed by ${ }^{3} \mathrm{H}$-thymidine incorporation, were inhibited by about $10-15 \%$ in presence of the remedies and by about $5 \%$ in presence of the solvent (data not shown).

Interestingly, the cytotoxic effect of two of the remedies investigated in this study, Carcinosin and Phytolacca, appeared similar to the activity of $0.12 \mu \mathrm{M}$ paclitaxel (Taxol), the most commonly used chemotherapeutic drug for breast cancer, when it was tested in the two adenocarcinoma cell lines investigated in this study in parallel experiments (data not shown). While it is significant that the remedies negatively affected the survival of the wild-type p53 and estrogen-responsive MCF-7 cells as well as p53-mutated and estrogen-independent MDA-MB-231 cells, the findings of differential susceptibility in these two cell types also indicate that the cytotoxic activities of the remedies are dependent on the genetic background of the treated cancer cells.

In this context, it is intriguing that a publication has reported lack of in vitro growth-inhibitory activity of the remedies investigated in this study in the breast adenocarcinoma cell line MDA-MB-231 and three additional prostate cancer cell lines, even though the same investigators had earlier reported significant anti-tumor activity of the remedies in an in vivo model of prostate cancer in Copenhagen rats (7). While these apparently paradoxical in vitro and in vivo results are somewhat puzzling, the results of this study provide a mechanistic explanation supporting the previously observed in vivo antitumor effects of these remedies reported by the other group. It is, however, worth mentioning that the in vitro study published by this group analyzed for gene expression alterations at the transcript level by RNAse protection assay, which is not expected to detect the changes in protein expression profiles of the genes regulating cell death, observed in this investigation.

Transition through the cell cycle phases is regulated by CDKs in complex with the specific cyclins (8). Cyclin D1 and D3 interact with their regulatory subunits, CDK4 and CDK6, and the active kinases phosphorylate the $\mathrm{Rb}$ protein to release the transcription factors of the E2F family, which transactivate the genes regulating G1-S phase transition. The fact that the remedy-treated HMLE and MCF-7 cells showed marked downregulation of phosphorylated $\mathrm{Rb}$ with accompanying changes in the cyclin and CDK levels demonstrates that the remedies interfere with the cell cycle regulatory pathways, causing G1 phase delay/arrest in these cells.

Regulation of cell cycle progression is also controlled by well-coordinated inactivation of the CDKs $(9,10)$. The members of the CDK inhibitor family of proteins, such as p21 and p27, are known to inactivate the cyclin/CDK complexes to regulate G1-S and G2-M phase transitions $(8,11)$. Both p21 and p27 physically interact with the CDKs at the amino terminal domain to inhibit their kinase activity and in the event of genomic damage activate the checkpoint response pathways by preventing the cells from initiating DNA replication and mitosis. This helps maintain genomic integrity $(12,13)$. Additionally, there are published reports of p27 overexpression inducing apoptosis in various epithelial cancer cell lines (14). It is relevant in this context that cell cycle delay/arrest and the induction of the apoptotic cascade, evident from the presence of reduced telomeric DNA (15) and PARP cleavage as well as activated caspase 7 in the remedy-treated carcinoma cells, were accompanied by upregulation of p27. Inactivation of CDK inhibitors, commonly observed in cancer, is believed to endow the tumor cells with endless survival and proliferation potential. Thus, it seems logical that upregulation of CDK inhibitors would 
activate the checkpoint response mechanisms and control survival and proliferation in cancer cells (16). Chemopreventive agents have also been described as having similar effects on the regulation of CDK inhibitors to induce proapoptotic response (17). Consistent with these findings and in view of our current observations, the ultra-diluted natural homeopathic remedies investigated in this study offer the promise of being effective preventive and/or therapeutic agents for breast cancer and worthy of further study.

In summary, our study demonstrates that the ultra-diluted natural product remedies prescribed in the 'Banerji Protocol' induce cell cycle delay/arrest with subsequent apoptosis in breast adenocarcinoma cells. Though the degree of the antisurvival effect appeared to correlate with the presence of the wild-type p53 gene, overall susceptibility to the inhibitory effects of the remedies appeared independent of the functional p53 and estrogen-receptor status of the breast carcinoma cells. Finally, the preferentially elevated cytotoxic effects on breast adenocarcinoma cells compared with cells derived from normal mammary epithelium raises the exciting possibility of a window of therapeutic opportunity for preferentially eliminating breast cancer cells with minimal damage to the surrounding normal mammary tissue by using the ultra-diluted remedies investigated in this report. The findings of this study should encourage further preclinical and animal investigation of these remedies as preventive and/ or therapeutic treatments for breast cancer.

\section{Acknowledgements}

The study was wholly supported by internal funds of MD Anderson Cancer Center. We also acknowledge Zhijun Liu, $\mathrm{Ph} . \mathrm{D}$ at Louisana State University for the constituent analysis of the ultra diluted remedies.

\section{References}

1. Barnes P, Bloom B and Nahin R: Complementary and alternative medicine use among adults and children: United States, 2007. National Health Statistics Reports; no. 12 National Center for Health Statistics, Hyattsville, MD, 2008.

2. Johannessen $\mathrm{H}$, von Bornemann Hjelmborg J, Pasquarelli E, Fiorentini G, Di Costanzos F and Miccinesi G: Prevalence in the use of complementary medicine among cancer patients in Tuscany, Italy. Tumori 94: 406-410, 2008.
3. Trager-Maury S, Tournigand C, Maindrault-Goebel F, Afchain P, de Gramont A, Garcia-Larnicol ML, Gervais H and Louvet C: Use of complementary medicine by cancer patients in a French oncology department. Bull Cancer 94: 1017-1025, 2007.

4. Banerji P and Campbell DR: Cancer patients treated with the Banerji protocols utilising homoeopathic medicine: A Best Case Series Program of the National Cancer Institute USA. Oncol Rep 20: 69-74, 2008.

5. Banerji P: P. Banerji Homeopathic Research Foundation (PBHRF) in Kolkata, India, 2008.

6. Pathak S, Multani AS and Banerji P: Ruta 6 selectively induces cell death in brain cancer cells but proliferation in normal peripheral blood lymphocytes: a novel treatment for human brain cancer. Int J Oncol 23: 975-982, 2003.

7. Thangapazham RL, Gaddipati JP, Rajeshkumar NV, Sharma A, Singh AK, Ives JA, Maheshwari RK and Jonas WB: Homeopathic medicines do not alter growth and gene expression in prostate and breast cancer cells in vitro. Integr Cancer Ther 5: 356-361, 2006.

8. Sherr CJ and Roberts JM: CDK inhibitors: positive and negative regulators of G1-phase progression. Genes Dev 13: 1501-1512, 1999.

9. Shapiro GI: Cyclin-dependent kinase pathways as targets for cancer treatment. J Clin Oncol 24: 1770-1783, 2006.

10. Lee YM and Sicinski P: Targeting cyclins and cyclin-dependent kinases in cancer: lessons from mice, hopes for therapeutic applications in human. Cell Cycle 5: 2110-2114, 2006.

11. Spankuch B, Kurunci-Csacsko E, Kaufmann M and Strebhardt K: Rational combinations of siRNAs targeting Plk1 with breast cancer drugs. Oncogene 26: 5793-5807, 2007.

12. Schwartz GK and Shah MA: Targeting the cell cycle: a new approach to cancer therapy. J Clin Oncol 23: 9408-9421, 2005.

13. Enders GH and Maude SL: Traffic safety for the cell: influence of cyclin-dependent kinase activity on genomic stability. Gene 371: 1-6, 2006.

14. Wang X, Gorospe M, Huang Y and Holbrook NJ: p27Kip1 overexpression causes apoptotic death of mammalian cells. Oncogene 15: 2991-2997, 1997.

15. Pathak S, Multani AS, Narayan S, Kumar V and Newman RA: Anvirzel, an extract of Nerium oleander, induces cell death in human but not murine cancer cells. Anticancer Drugs 11: 455-463, 2000.

16. Roy S, Gu M, Ramasamy K, Singh RP, Agarwal C, Siriwardana S, Sclafani RA and Agarwal R: p21/Cip1 and p27/Kip 1 are essential molecular targets of inositol hexaphosphate for its antitumor efficacy against prostate cancer. Cancer Res 69: 1166-1173, 2009.

17. Singh RP and Agarwal R: Natural flavonoids targeting deregulated cell cycle progression in cancer cells. Curr Drug Targets 7: 345-354, 2006. 